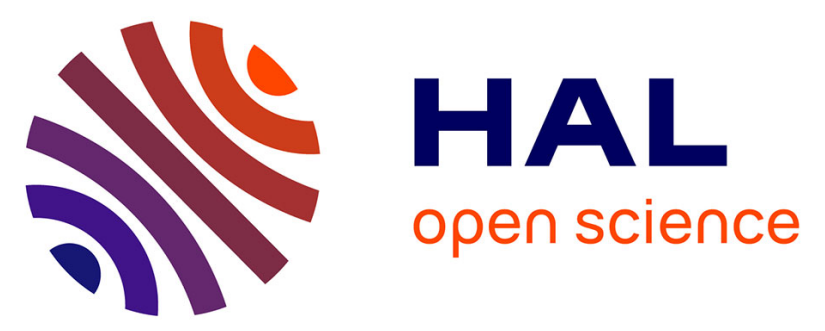

\title{
Random Germs and Stochastic Watershed for Unsupervised Multispectral Image Segmentation
}

Guillaume Noyel, Jesus Angulo, Dominique Jeulin

\section{To cite this version:}

Guillaume Noyel, Jesus Angulo, Dominique Jeulin. Random Germs and Stochastic Watershed for Unsupervised Multispectral Image Segmentation. 11th International Conference Knowledge-Based Intelligent Information and Engineering Systems (KES 2007), Sep 2007, Vietri sul Mare, Salerno, Italy. pp.17-24, 10.1007/978-3-540-74829-8_3 . hal-01263963

\section{HAL Id: hal-01263963 https://hal.science/hal-01263963}

Submitted on 29 Jan 2016

HAL is a multi-disciplinary open access archive for the deposit and dissemination of scientific research documents, whether they are published or not. The documents may come from teaching and research institutions in France or abroad, or from public or private research centers.
L'archive ouverte pluridisciplinaire HAL, est destinée au dépôt et à la diffusion de documents scientifiques de niveau recherche, publiés ou non, émanant des établissements d'enseignement et de recherche français ou étrangers, des laboratoires publics ou privés.

$$
\text { Copyright }
$$




\title{
Random Germs and Stochastic Watershed for Unsupervised Multispectral Image Segmentation
}

\author{
Guillaume Noyel, Jesús Angulo, Dominique Jeulin \\ Centre de Morphologie Mathématique, Ecole des Mines de Paris \\ 35 rue Saint Honoré, 77305 Fontainebleau, France \\ \{guillaume.noyel, jesus . angulo, dominique.jeulin\}@ensmp.fr
}

\begin{abstract}
This paper extends the use of stochastic watershed, recently introduced by Angulo and Jeulin [1], to unsupervised segmentation of multispectral images. Several probability density functions (pdf), derived from Monte Carlo simulations ( $M$ realizations of $N$ rsandom markers), are used as a gradient for segmentation: a weighted marginal pdf a vectorial pdf and a probabilistic gradient. These gradient-like functions are then segmented by a volume-based watershed algorithm to define the $R$ largest regions. The various gradients are computed in multispectral image space and in factor image space, which gives the best segmentation. Results are presented on PLEIADES satellite simulated images.
\end{abstract}

Key words: multispectral image, unsupervised segmentation, mathematical morphology, stochastic watershed

\section{Introduction}

Watershed transformation is one of the most powerful tools for image segmentation. Starting from a gradient, the classical paradigm of watershed segmentation consists in determining markers for each region of interest. The markers avoid the over-segmentation as a region is associated to each marker. When the markers cannot be easily defined several hierarchical approaches exist. They are mainly divided in two hierarchical techniques: 1) non-parametric waterfalls algorithm [2] and 2) hierarchies based on extinction values, which allow to select the minima used in the watershed according to morphological criteria (dynamics, surface and volume) $[6,8]$. Usually, the volume criteria is the most powerful.

In [1], Angulo and Jeulin, defined a new method: stochastic watershed. In the present paper, the method involving Monte Carlo simulation on the image is extended to multispectral images.

\section{Random germs and stochastic watershed segmentation}

One of the main artefacts of classical watershed is that small regions strongly depend on the position of the markers, or on the volume, i.e. the integral of the gray levels of the catchment basin, associated to their minima. In fact, there 
are two kinds of contours associated to the watershed of a gradient: 1st order contours, which correspond to significant regions and which are relatively independent from markers; and 2nd order contours, associated to "small", "low" contrasted or textured regions, which depend strongly on the location of markers. Stochastic watershed aims at enhancing the 1st order contours from a sampling effect, to improve the result of watershed.

Let us consider $\left\{m r k_{i}(x)\right\}_{i=1}^{M}$ a series of $M$ realizations of $N$ uniform or regionalized random germs. Each one of these binary images is considered as the marker for a watershed segmentation of a scalar gradient or a vector gradient. Therefore, a series of segmentations is obtained, i.e. $\left\{s g_{i}^{m r k}(x)\right\}_{i=1}^{M}$. Starting from the $M$ realizations of contours, the probability density function $p d f(x)$ of contours is computed by the Parzen window method [9]. The smoothing effect of the Gaussian convolution kernel (typically $\sigma=3$ working on contours of one pixel width) is important to obtain a function where closed contours, such as textured regions or associated to small regions, are added together. The $p d f(x)$ is thresholded to obtain the most prominent contours.

Moreover, we segment the $\operatorname{pdf}(x)$ image, using a watershed segmentation with a volume criteria in order to select the largest regions.

\section{Multispectral image space (MIS) vs. factor image space (FIS)}

In the current paper, multispectral images are used. Multispectral images are multivariate discrete functions with typically fewer than ten bands. In a formal way, in each pixel of a 2D multispectral image is considered a vector with values in wavelength. To each wavelength corresponds an image in two dimensions called channel. The number of channels depends on the nature of the specific problem under study (satellite imaging, microscopic images, etc).

Let $\mathbf{f}_{\lambda}: E \rightarrow \mathcal{T}^{L}\left(x \rightarrow \mathbf{f}_{\lambda}(x)=\left(f_{\lambda_{1}}(x), f_{\lambda_{2}}(x), \ldots, f_{\lambda_{L}}(x)\right)\right)$, be a multispectral image, where: $E \subset \mathbb{R}^{2}, \mathcal{T} \subset \mathbb{R}$ and $\mathcal{T}^{L}=\mathcal{T} \times \mathcal{T} \times \ldots \times \mathcal{T} ; x=x_{i} \backslash i \in$ $\{1,2, \ldots, P\}$ is the spatial coordinates of a vector pixel $\mathbf{f}_{\lambda}\left(x_{i}\right)$ ( $P$ is the pixels number of $E) ; f_{\lambda_{j}} \backslash j \in\{1,2, \ldots, L\}$ is a channel ( $L$ is the channels number); $f_{\lambda_{j}}\left(x_{i}\right)$ is the value of vector pixel $\mathbf{f}_{\lambda}\left(x_{i}\right)$ on channel $f_{\lambda_{j}}$.

Due to the redundancy of channels, a data reduction is usually performed using Factor Correspondence Analysis (FCA) [3]. We prefer a FCA in place of a Principal Component Analysis (PCA), because image values are positive and the spectral channels can be considered as probability distributions. As for PCA, from selected factorial FCA axes the image can be partially reconstructed. The metric used in FCA is the chi-squared, which is adapted to probability laws and normalized by channels weight. FCA can be seen as a transformation going from image space to factorial space. In factorial space the coordinates of the pixels vector on each factorial axis are called pixels factors. The pixels factors can be considered as another multispectral image whose channels correspond to factorial axes: $\zeta: \mathcal{T}^{L} \rightarrow \mathcal{T}^{K} / K<L\left(\mathbf{f}_{\lambda}(x) \rightarrow \mathbf{c}_{\alpha}^{\mathbf{f}}(x)=\left(c_{\alpha_{1}}^{\mathbf{f}}(x), \ldots, c_{\alpha_{K}}^{\mathbf{f}}(x)\right)\right)$ 
A limited number $K$ of factorial axes is usually chosen. It is smaller than the channels number $L$. Therefore FCA can be seen as a projection of initial vector pixels in a factor space with a lower dimension. Moreover FCA can be used to reduce noise in the image [5,7]. Consequently, we have two spaces for the multivariate segmentation: the multispectral image space (MIS) and the factor image space (FIS). Fig. 1 gives an example of a five bands satellite simulated image PLEIADES, acquired by the CNES (Centre National d'Etudes Spatiales, the French space agency) and provided by Flouzat [4], and its corresponding FCA representation. Its channels are the following: $f_{\lambda_{1}}$ blue, $f_{\lambda_{2}}$ green, $f_{\lambda_{3}}$ red, $f_{\lambda_{4}}$ near infrared and $f_{\lambda_{5}}$ panchromatic. The panchromatic channel, initially of size $1460 \times 1460$ pixels with a resolution of 0.70 meters, was resized to $365 \times 365$ pixels. Therefore, the resolution is 2.80 meters in an image of $365 \times 365 \times 5$ pixels. In order to represent a multispectral image in a synthetic way, we have created a synthetic RGB image using channels $f_{\lambda_{3}}$ red, $f_{\lambda_{2}}$ green and $f_{\lambda_{1}}$ blue.

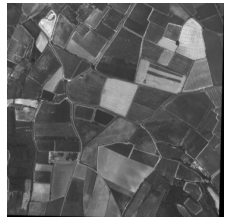

$\mathbf{f}_{\lambda_{1}}$ blue Copyright (C)

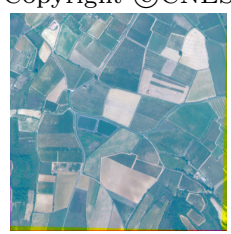

Synthetic RGB

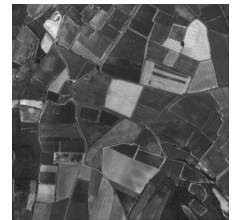

$\mathbf{f}_{\lambda_{2}}$ green

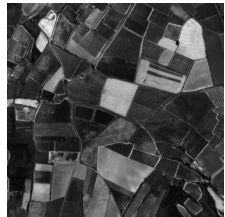

$\mathbf{f}_{\lambda_{3}}$ red

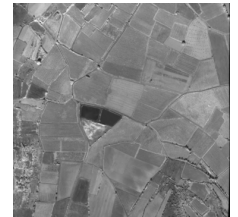

$\mathbf{f}_{\lambda_{4}}$ near inf.-red $\mathbf{f}_{\lambda_{5}}$ panchrom.

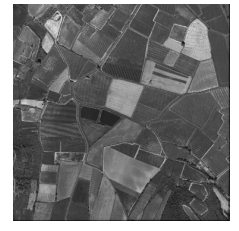

Copyright (C) CNES

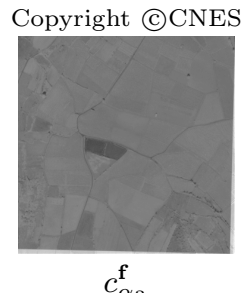

Fig. 1. Channels of multispectral image $\mathbf{f}_{\lambda}$ "Roujan", synthetic RGB representation, FCA factors pixels $\mathbf{c}_{\alpha}^{\mathbf{f}}$ on axes 1,2 and 3 with respective inertias $84.1 \%, 8.7 \%, 6.2 \%$

\section{Spectral distances and gradients for MIS and FIS}

In order to segment images according to watershed-based paradigms, a gradient is needed. Intuitively, a gradient is an image which quantifies the values of contours between regions. A gradient image, in fact the norm, is a scalar function with values in the reduced interval $[0,1]$, i.e. $\varrho(x): E \rightarrow[0,1]$. In order to define a gradient, two approaches are considered: the standard symetric morphological gradient on each marginal channel and a metric-based vectorial gradient on all channels. The morphological gradient can only be applied on scalar images and is defined as the difference between dilation and erosion with 
a unit structuring element $B$ [2], i.e. $\varrho\left(f_{\lambda_{j}}(x)\right)=\delta_{B}\left(f_{\lambda_{j}}(x)\right)-\varepsilon_{B}\left(f_{\lambda_{j}}(x)\right)$. The metric-based gradient is a vectorial gradient defined as the difference between the supremum and the infimum of a defined distance on a unit neighbourhood $B(x): \varrho^{d}\left(\mathbf{f}_{\lambda}\right)(x)=\vee\left[d\left(\mathbf{f}_{\lambda}(x), \mathbf{f}_{\lambda}(y)\right), y \in B(x)\right]-\wedge\left[d\left(\mathbf{f}_{\lambda}(x), \mathbf{f}_{\lambda}(y)\right), y \in B(x)\right]$, where $d: \mathcal{T}^{L} \times \mathcal{T}^{L} \rightarrow[0, \infty[$ is the metric distance used to calculate the difference between points in the neighborhood $B$. Various metric distances, useful for multispectral images, are available for this gradient such as: the Euclidean distance: $d_{E}\left(\mathbf{f}_{\lambda}(x), \mathbf{f}_{\lambda}(y)\right)=\sqrt{\sum_{j=1}^{L}\left(f_{\lambda_{j}}(x)-f_{\lambda_{j}}(y)\right)^{2}}$ and the Chisquared distance: $d_{\chi^{2}}\left(\mathbf{f}_{\lambda}\left(x_{i}\right), \mathbf{f}_{\lambda}\left(x_{i^{\prime}}\right)\right)=\sqrt{\sum_{j=1}^{L} \frac{S}{f_{. \lambda_{j}}}\left(\frac{f_{\lambda_{j}}\left(x_{i}\right)}{f_{x_{i}}}-\frac{f_{\lambda_{j}}\left(x_{i^{\prime}}\right)}{f_{x_{i^{\prime}}}}\right)^{2}}$ with $f_{. \lambda_{j}}=\sum_{i=1}^{P} f_{\lambda_{j}}\left(x_{i}\right), f_{x_{i} .}=\sum_{j=1}^{L} f_{\lambda_{j}}\left(x_{i}\right)$ and $S=\sum_{j=1}^{L} \sum_{i=1}^{P} f_{\lambda_{j}}\left(x_{i}\right)$.

Besides, marginal gradients from spectral bands can be combined (by addition or supremum) to define new gradients. If all factor axes are kept, the chi-squared distance in MIS is equivalent to the Euclidian distance in FIS [3]. Therefore, the vector metric-based gradient is more appropriate in MIS, with the chi-squared distance $d_{\chi^{2}}$ and in FIS, with the Euclidian distance $d_{E}$.

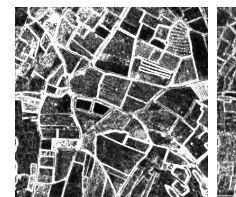

$\varrho\left(f_{\lambda_{3}}\right)$

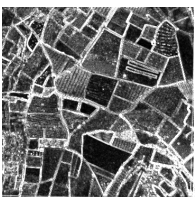

$\varrho^{\chi^{2}}\left(\mathbf{f}_{\lambda}\right)$

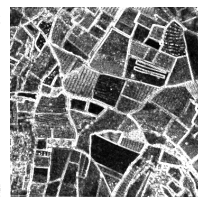

$\varrho^{E}\left(\mathbf{c}_{\alpha}^{\mathbf{f}}\right)$

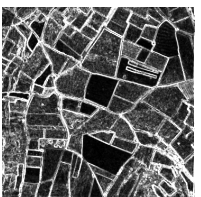

$\varrho\left(c_{\alpha_{1}}^{\mathbf{f}}\right)$

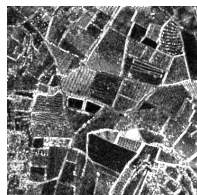

$\varrho\left(c_{\alpha_{2}}^{\mathbf{f}}\right)$

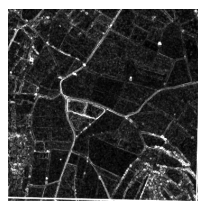

$\varrho\left(c_{\alpha_{3}}^{\mathbf{f}}\right)$

Fig. 2. Morphological gradient $\varrho\left(f_{\lambda_{3}}\right)$ on channel 3, metric-based vectorial gradient in image space $\varrho^{\chi^{2}}\left(\mathbf{f}_{\lambda}\right)$, the same in factor space $\varrho^{E}\left(\mathbf{c}_{\alpha}^{\mathbf{f}}\right)(3$ axes $)$ and morphological gradients of each components of factor space $\varrho\left(c_{\alpha_{1}}^{\mathbf{f}}\right), \varrho\left(c_{\alpha_{2}}^{\mathbf{f}}\right), \varrho\left(c_{\alpha_{3}}^{\mathbf{f}}\right)$.

In the example of figure 2 , we notice that the morphological gradients of the pixels factors on the axes $2, \varrho\left(c_{\alpha_{2}}^{\mathbf{f}}\right)$, and $3, \varrho\left(c_{\alpha_{3}}^{\mathbf{f}}\right)$, contain less information than the morphological gradient of the pixels factors on axis $1, \varrho\left(c_{\alpha_{1}}^{\mathbf{f}}\right)$. Besides, the inertia of axes 2 and $3,8.7 \%$ and $6.2 \%$ respectively, are very small. Therefore, we will only keep the first factor axis.

\section{$5 \quad$ Weighted marginal pdf, vectorial pdf and probabilistic vectorial gradient}

In the sequel, we define functions used as gradients $g$ for segmentation: the weighted marginal probability density function $m p d f$, the vectorial pdf $v p d f$ and the probabilistic vectorial gradient $\varrho_{\text {prob }}$. The weighted marginal pdf $m p d f$ is computed as follows:

- For the morphological gradient of each channel $\varrho\left(f_{\lambda_{j}}\right), j \in[1, \ldots, L]$, throw $M$ realizations of $N$ uniform random germs, i.e. the markers $\left\{m r k_{i}^{j}\right\}_{i=1 \ldots M}^{j=1 \ldots L}$, 
generating $M \times L$ realizations. Get the series of segmentations, $\left\{s g_{i}^{j}(x)\right\}_{i=1 \ldots M}^{j=1 \ldots L}$, by watershed associated to morphological gradients of each channel $\varrho\left(f_{\lambda_{j}}\right)$.

- Get the marginal pdf on each channel $p d f_{j}(x)=\frac{1}{M} \sum_{i=1}^{M} s g_{i}^{j}(x) * G_{\sigma}$.

- Obtain the weighted marginal pdf $\operatorname{mpdf}(x)=\sum_{j=1}^{L} w_{j} p d f_{j}(x)$, with $w_{j}=$ $1 / L, j \in[1, \ldots, L]$ in MIS and $w_{j}$ equal to the inertia axes in FIS.

The vectorial pdf $v p d f$ is obtained as follows:

- For the vectorial gradient $\varrho^{d}\left(\mathbf{f}_{\lambda}\right)$, throw $M \times L$ realizations of $N$ uniform random germs, i.e. the markers $\left\{m r k_{i}\right\}_{i=1 \ldots M \times L}$, with $L$ the channels number. Get the segmentation, $\left\{s g_{i}(x)\right\}_{i=1 \ldots M \times L}$, by watershed associated to the vectorial gradient $\varrho^{d}\left(\mathbf{f}_{\lambda}\right)$, with $d=d_{\chi^{2}}$ in MIS or $d=d_{E}$ in FIS.

- Obtain the probability density function $\operatorname{vpdf}(x)=\frac{1}{M \times L} \sum_{i=1}^{M \times L} s g_{i}(x) * G_{\sigma}$.

The probabilistic vectorial gradient is defined as $\varrho_{\text {prob }}=m p d f+\varrho^{d}$ : after normalization in $[0,1]$ of the weighted marginal pdf mpdf and the metric-based gradient $\varrho^{d}$.

After computing them, these three functions are used as a gradient $g$ of a new watershed with volume constraint $R$, i.e. the $R$ regions with the largest volume, to obtain the segmentation contours of the image.

\section{Application: unsupervised segmentation of multi-band satellite images}

We compare 4 methods of segmentation in MIS based on the volume-based watershed on functions used as a gradient $g . g$ takes several values, giving the associated watershed segmentations, such as: the chi-squared metric based gradient $\varrho^{\chi^{2}}: \operatorname{seg}^{\text {vol }}\left(\varrho^{\chi^{2}}\left(\mathbf{f}_{\lambda}\right), R\right)$ (the reference segmentation), the weighted marginal pdf $m p d f: \operatorname{seg}^{v o l}\left(\operatorname{mpdf}\left(\mathbf{f}_{\lambda}\right), R\right)$, the vectorial pdf $v p d f: \operatorname{seg}^{v o l}\left(v p d f\left(\mathbf{f}_{\lambda}\right), R\right)$, and the probabilistic gradient $\varrho_{\text {prob }}: \operatorname{seg}^{v o l}\left(\varrho_{\text {prob }}\left(\mathbf{f}_{\lambda}\right), R\right)$. The results are presented in synthetic RGB images made with channels $f_{\lambda_{3}}$ red, $f_{\lambda_{2}}$ green and $f_{\lambda_{1}}$ blue. Besides, more details are available in [1] about the execution time and the influence of the three parameters on the number: of realizations $M$, of regions $R$ and of germs $N$.

In figure 3 , we notice that the segmentation results are much better (i.e. more homogenous in space) for the watershed on probabilistic functions (mpdf, $v p d f$, $\left.\varrho_{\text {prob }}\right)$ than on the standard vector gradient $\varrho^{\chi^{2}}$, which is used for the classical watershed. Therefore the stochastic watershed is better than the classical one. Besides, the probabilistic gradient $\varrho_{\text {prob }}$, which combines a vectorial gradient $\varrho^{d}$ and the weighted marginal pdf $m p d f$, is adapted to segment the 1st order contours (the largest regions) and recover some of the 2nd order contours (the "small", "low" contrasted or textured regions).

Moreover, we have tested in the factor image space (FIS) the watershed with a volume constraint (fig. 4). As explained before, we will only use the first factor axis. As we keep only the first factor axis, the Euclidian metric based 


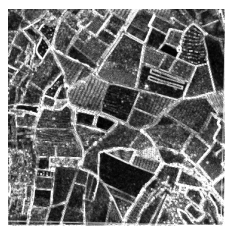

$\varrho^{\chi^{2}}\left(\mathbf{f}_{\lambda}\right)$

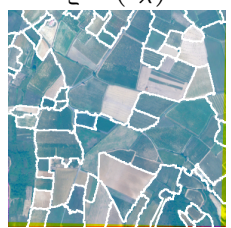$$
\text { - }
$$

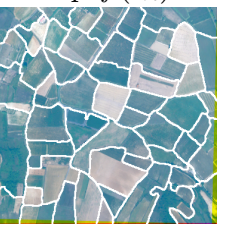

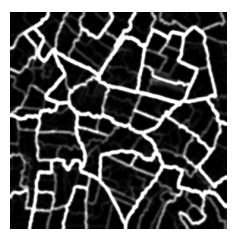

$\operatorname{vpdf}\left(\mathbf{f}_{\lambda}\right)$
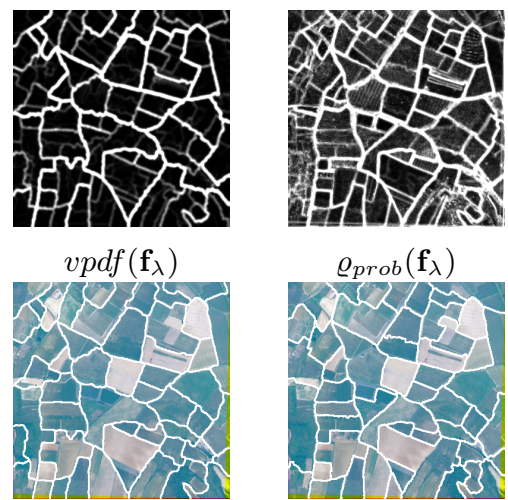

$\varrho_{\text {prob }}\left(\mathbf{f}_{\lambda}\right)$

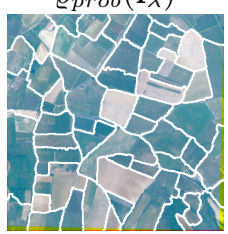

$\operatorname{seg}^{\text {vol }}\left(\varrho_{\text {prob }}\left(\mathbf{f}_{\lambda}\right), R\right)$

Fig. 3. Top: Gradients and pdf; bottom: associated watershed segmentations on image "Roujan", in MIS, with $N=50$ points , $M=100$ realizations, $R=50$ regions.

gradient $\varrho^{E}$ is very close to the morphological gradient. We compute the watershed segmentation on the functions used as gradients $g$ : the morphological gradient $\varrho: \operatorname{seg}^{v o l}\left(\varrho\left(c_{\alpha_{1}}^{\mathbf{f}}\right), R\right)$, the weighted marginal pdf $m p d f$ (i.e; the morphological gradient pdf): $\operatorname{seg}^{v o l}\left(m p d f\left(c_{\alpha_{1}}^{\mathrm{f}}\right), R\right)$ and the probabilistic gradient $\varrho_{\text {prob }}$ : $\operatorname{seg}^{v o l}\left(\varrho_{\text {prob }}\left(c_{\alpha_{1}}^{\mathrm{f}}\right), R\right)$.

We notice that the contours on morphological gradient $\varrho$ are comparable to those on weighted marginal pdf $m p d f$ and on probabilistic gradient $\varrho_{\text {prob }}$. In fact, due to FCA, the noise is rejected on the factor axes of smaller inertia $[5,7]$. However segmentations of 1 st order contours on mpdf and $\varrho_{p r o b}$ visually seems a bit better than on $\varrho$. Therefore, working on the firsts axes improves the segmentation as they contain less noise.
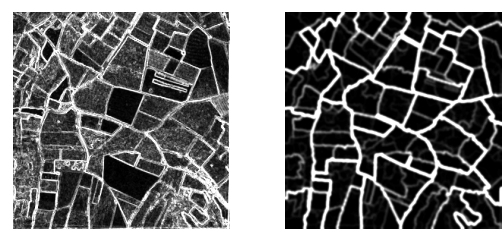

$\varrho\left(c_{\alpha_{1}}^{\mathbf{f}}\right)$
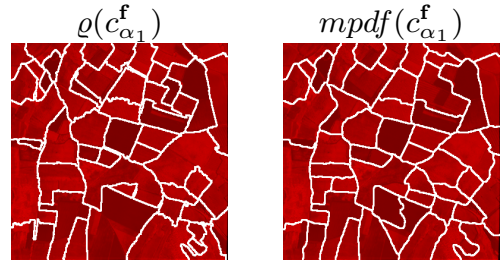

$$
\operatorname{seg}^{v o l}\left(\varrho\left(c_{\alpha_{1}}^{\mathbf{f}}\right), R\right) \operatorname{seg}^{2}
$$
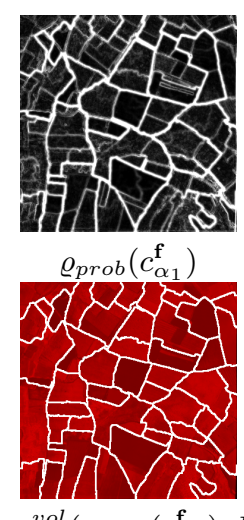

Fig. 4. Segmentation watershed in FIS with $N=50$ points, $M=100$ realizations and $R=50$ regions. 
Besides, by observing several segmentation results in various images (fig. 5), we notice that segmentations on weighted marginal pdf $m p d f$ have contours more regular than on vectorial pdf $v p d f$. Consequently, the weighted marginal pdf mpdf seems to be the best approach for extracting area with smooth contours, like roads, buildings, fields, ... in satellite images.

\section{Conclusions and perspectives}

We have shown the interest of using stochastic watershed segmentation to unsupervised segment multispectral images. Several variants are available to calculate the functions used as gradient $g$ for the watershed segmentation: a weighted marginal pdf $m p d f$, a vectorial pdf $v p d f$ or a probabilistic gradient $\varrho_{\text {prob }}$. These gradients like functions are used for stochastic watershed to improve the classical watershed applied on a vector gradient. In fact, the weighted marginal pdf gives the best segmentation results and the probabilistic gradient is useful to find the principal and the secondary contours. Moreover, working in factorial image space FIS is generally better for segmentation than working in multispectral image space MIS. In the future we are thinking of using regionalized germs to build the pdf in order to segment a target in an image.

Acknowledgments: The authors are grateful to Prof. Guy Flouzat (Laboratoire de Télédétection à Haute Résolution, LTHR/ ERT 43 / UPS, Université Paul Sabatier, Toulouse 3) for his collaboration on PLEIADES satellite simulated images in the framework of ORFEO program (Centre National d'Etudes Spatiales, the French space agency).

\section{References}

1. Angulo, J., Jeulin, D.: Stochastic watershed segmentation. Submit. to Int. Symp. Mathematical Morphology (ISMM'07) Rio, Brazil, October 10-13 (2007)

2. Beucher, S.: Watershed, hierarchical segmentation and waterfall algorithm, Proc. Int. Symp. Mathematical Morphology ISMM'94 (1994) 69-76

3. Benzécri, J.P.: L'Analyse Des Données. L'Analyse des Correspondances, Vol. 2, Paris Dunod (1973) 1-166.

4. Laporterie-Déjean, F, De Boissezon, H., Flouzat, G., Lefèvre-Fonollosa, M.J.: Thematic and statistical evaluations of five panchromatic/multispectral fusion methods on simulated PLEIADES-HR images, Information Fusion Vol. 6 (2005) 193-212.

5. Green, A.A., Berman, M., Switzer, P., Craig, M.D.: A Transformation for Ordering Multispectral Data in terms of Image Quality with Implications for Noise Removal, IEEE Trans. Geosc. Rem. Sens. Vol. 26 (1988) 65-74.

6. Meyer, F.: An overview of Moprhological Segmentation, International Journal of Pattern Recognition and Artificial Intelligence, Vol. 15 (2001), no.7, 1089-1118

7. Noyel, G., Angulo, J., Jeulin, D.: Morphological Segmentation of hyperspectral images, Submitted to Image Analysis and Stereology, ICS XII St Etienne Aug. 30 Sept. 7 2007, Internal notes Ecole des Mines de Paris no. N-36/06/MM (2007)

8. Vachier, C., Meyer, F.: Extinction value: a new measurement of persistence, IEEE Worksop on Nonlinear Signal and Image Processing (1995) 254-257

9. Duda, R.O., Hart, P.E.: Pattern Classification and Scene Analysis, Wiley (1973) 

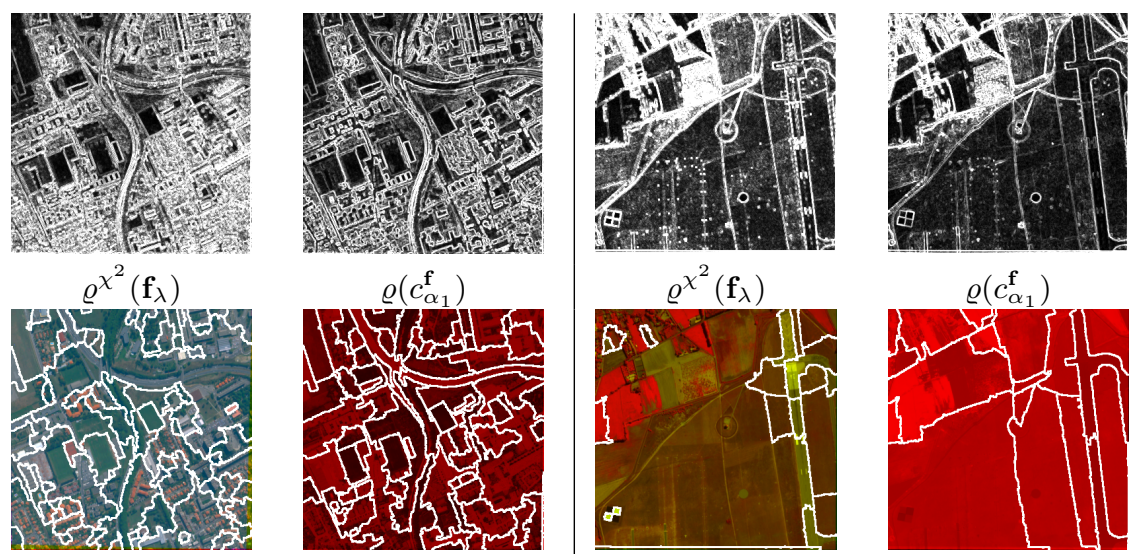

$\operatorname{seg}^{\text {vol }}\left(\varrho^{\chi^{2}}\left(\mathbf{f}_{\lambda}\right), R\right)$
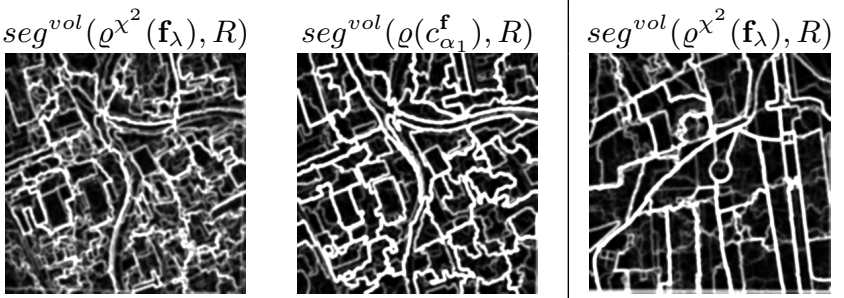

$\operatorname{seg}^{v o l}\left(\varrho\left(c_{\alpha_{1}}^{\mathbf{f}}\right), R\right)$

$\operatorname{mpdf}\left(\mathbf{f}_{\lambda}\right)$ $\operatorname{mpdf}\left(c_{\alpha_{1}}^{\mathbf{f}}\right)$
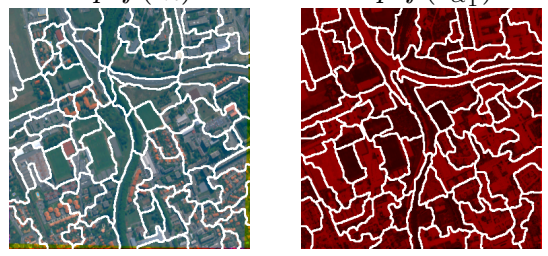

$\operatorname{mpdf}\left(\mathbf{f}_{\lambda}\right)$
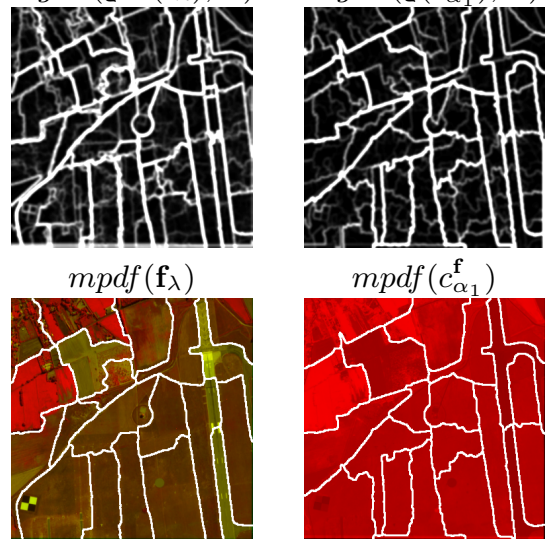

$\operatorname{mpdf}\left(c_{\alpha_{1}}^{\mathbf{f}}\right)$

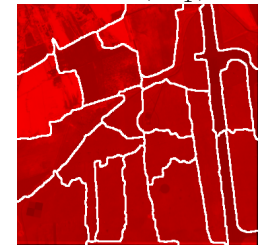

$\operatorname{seg}^{v o l}\left(\operatorname{mpdf}\left(\mathbf{f}_{\lambda}\right), R\right) \operatorname{seg}^{v o l}\left(\operatorname{mpdf}\left(c_{\alpha_{1}}^{\mathbf{f}}\right), R\right)$

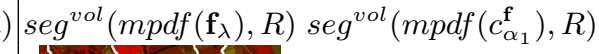

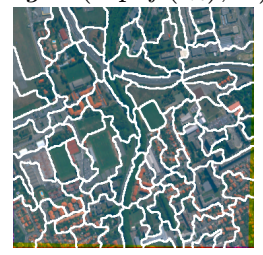

$\operatorname{seg}^{v o l}\left(v p d f\left(\mathbf{f}_{\lambda}\right), R\right)$

(a) (b)

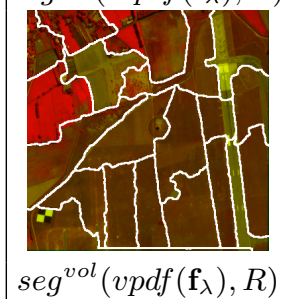

(c) (d)

Fig. 5. Watershed segmentations and associated gradients like functions in MIS or FIS on other images. (a) image "Toulouse" ( 5 channels) $\mathrm{N}=100, \mathrm{M}=100, \mathrm{R}=75$ (b) Factor axis $1 c_{\alpha_{1}}^{\mathrm{f}}$ (inertia 77.4\%) of image "Toulouse" (c) "Salon de Provence" (4 channels) N= $50, \mathrm{M}=100, \mathrm{R}=20(\mathrm{~d})$ Factor axis $1 c_{\alpha_{1}}^{\mathrm{f}}$ (inertia $82.2 \%$ ) of image "Salon de Provence". 